\title{
Getting your Scanning Electron Microscope to Perform at Atomic Resolution Levels
}

\author{
Andras Vladar and Kerim Arat
}

Natl. Inst. of Standards and Technology, Gaithersburg, Maryland, United States

In addition to being a key instrument in the hundreds of billions of dollar/year semiconductor-, nano-, and bio industries, the scanning electron microscope (SEM) is indispensable for the advancement of science and technology. Modern SEMs are capable of imaging with spatial resolution down to sub-0.5 nm, essentially at atomic levels, and three-dimensional shape measurements of sub-10 nm structures with a few atoms worth of accuracy are also feasible.[1] Beyond atomic resolution capabilities, SEMs with cryogenic and environmental capabilities, massive numbers of parallel beams, and low and ultra-low landing energy operation are all commercially available and improving rapidly. Detector hardware advancements are also enabling a resurgence in scanning transmission electron microscopy in an SEM (STEM-in-SEM). This operation mode is rightfully gaining importance as people realize that for thin samples, low-Z samples, and samples susceptible to high-energy beam damage, SEMs can be as useful as traditional, high-landing-energy STEMs, and in some cases even better. [2,3]

Alas, the peak performance of SEMs is often hindered by a few common problems, and because of these problems it is likely that no SEM image or measurement result is ever created within fully optimal conditions. For example, it is well-understood that a stable environment/laboratory is important for optimal measurements, and improvements therein can often be implemented if needed. In many cases suboptimal results arise from lack of dependable focusing ability, uncompensated stage and beam drifts and ignored electron-beam-induced contamination [4] that is arising from the instrument and the sample. Fortunately, useful solutions for some of these problems exist, and new solutions that can lead to significant improvements are also being developed. For example, the figure below on the left shows that fine, nm-scale details become visible after electron cleaning of a slightly contaminated gold-on-carbon resolution sample.

In addition to physical environment and instrumental improvements, computational SEM using Monte Carlo simulations based on sophisticated physics models is particularly useful, both for determining optimal measurement conditions and for analysis of the acquired raw data to generate accurate physicsbased results with measurement uncertainties down to atomic levels. For example, besides operating the SEM at optimal performance, with the advent of high-resolution, low-landing energy STEM operation it is important to explore and understand the physics limits of the signals. Moreover, simulations can provide direction to optimize SEM operating conditions that provide the desired information in the most efficient manner. For example, the figure below on the right shows an example of simulated SEM images of a 60 $\mathrm{nm} \mathrm{Au}$ particle on a Si substrate. By combining simulations from different imaging modes, appropriate imaging mode conditions can be selected for the desired information, and how those imaging modes can be used for accurate three-dimensional reconstruction of different structures.

In this presentation we address the most bothersome problems of SEMs and demonstrate the possibility for significant improvements, even with older instruments. Examples for feasible future performance enhancements along with examples for simulation-based measurement optimization are also provided. With the application of the presented solutions it is possible to meet or beat the peak performance parameters of the SEM and generate excellent results any time. 

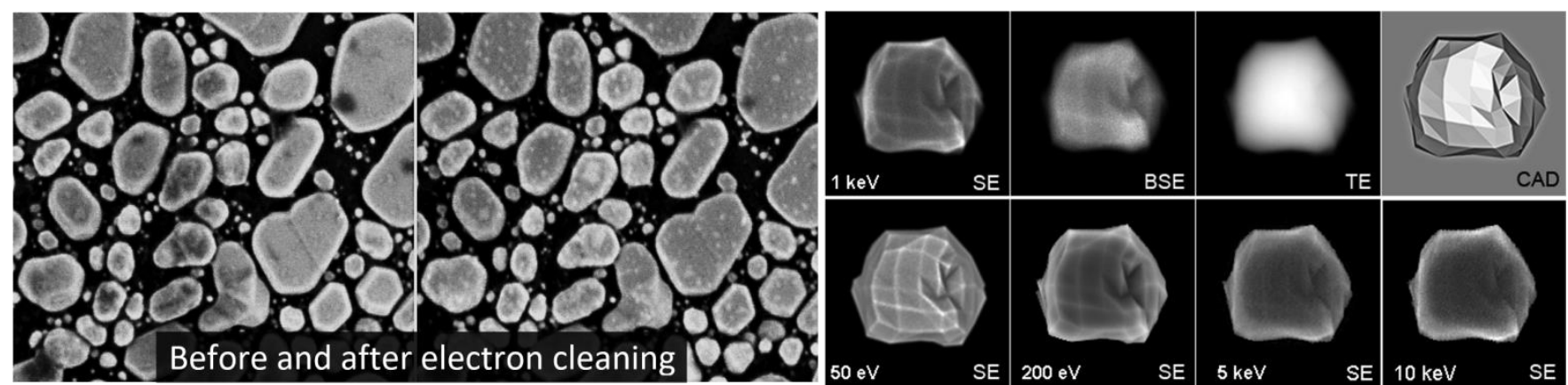

Figure 1. Left: ultra-high cleanliness results in better imaging resolution and makes atomic-level SEM measurements possible. Right: computational 3D nm-scale SEM metrology, prediction of likely images of nanostructures

\section{References}

1. $10 \mathrm{~nm}$ Three-dimensional CD-SEM Metrology, A. E. Vladár et al., https://doi.org/10.1117/12.2045977

2. Quantitative Comparison of simulated and measured signals in the STEM mode of a SEM, C.G.H. Walker et al. https://doi.org/10.1016/j.nimb.2017.10.034

3. Scattering intensity distribution dependence on collection angles in annular dark-field STEM-in-SEM images, J. Holm https://doi.org/10.1016/j.ultramic.2018.06.007

4. Electron Irradiation Cleaning of the SEM and its Samples, A.E. Vladár, et al. M\&M 2021 